Boris Utsch • John A. Sayer - Massimo Attanasio •

Rob Rodrigues Pereira - Michael Eccles •

Hans-Christian Hennies • Edgar A. Otto •

Friedhelm Hildebrandt

\title{
Identification of the first AHI1 gene mutations in nephronophthisis-associated Joubert syndrome
}

Received: 10 June 2005 / Revised: 5 July 2005 / Accepted: 5 July 2005 / Published online: 21 October 2005 (C) IPNA 2005

\begin{abstract}
Joubert syndrome (JBTS) is an autosomal recessive multisystem disease characterized by cerebellar vermis aplasia, mental retardation, muscular hypotonia, an irregular breathing pattern in the neonatal period and abnormal eye movements. Some individuals have progressive renal failure characterized by nephronophthisis (NPHP) and/or retinal dystrophy. Homozygous deletions of NPHP1 on chromosome $2 q 13$ have been identified in individuals with NPHP-associated JBTS. Recently, mutations in AHII on chromosome 6q23.3 were found in JBTS patients without NPHP. Here, by direct sequencing, we identify novel truncating mutations within AHII in affected patients from two families. One patient had the association of JBTS and NPHP with chronic renal failure. This is the first report of AHII mutations causing JBTS associated with NPHP, confirming the clinical and genetic heterogeneity of NPHP.
\end{abstract}

Boris Utsch and John A. Sayer contributed equally to this work

B. Utsch - J. A. Sayer - M. Attanasio - E. A. Otto · F. Hildebrandt Departments of Pediatrics and of Human Genetics,

University of Michigan, Ann Arbor, MI, USA

B. Utsch

Department of Pediatrics,

University Erlangen-Nuremberg, Erlangen, Germany

R. R. Pereira

Medical Center Rijnmond South,

Department of Pediatrics, Rotterdam, The Netherlands

M. Eccles

Developmental Genetics Laboratory,

Pathology Department, University of Otago, Otago, New Zealand

H.-C. Hennies

Gene Mapping Center, University of Cologne, Cologne, Germany

F. Hildebrandt $(\square)$

University of Michigan Health System,

8220C MSRB III, 1150 West Medical Center Drive,

Ann Arbor, MI, 48109-0646, USA

e-mail: fhilde@umich.edu

Tel.: +1-734-615 7285

Fax: +1-734-6151386
Keywords Joubert syndrome - Renal failure . Nephronophthisis $\cdot$ AHII $\cdot$ Mutational analysis

\section{Introduction}

Joubert syndrome (JBTS) is a rare autosomal recessive multisystem disorder characterized by aplasia of the cerebellar vermis, ataxia, abnormal eye movements, an abnormal breathing pattern in the neonatal period and psychomotor mental retardation [1, 2, 3]. On MRI brain imaging JBTS is characterized by a typical radiological finding, termed as "molar tooth sign" (MTS). This reflects a complex midbrain and hindbrain malformation consisting of an increased interpeduncular distance of the pontomesencephalic junction, cerebellar vermis aplasia and thickened, straight and elongated superior cerebellar peduncles $[4,5,6]$. The abnormal eye movements may comprise oculomotor apraxia (OMA) type Cogan, or difficulty with smooth eye pursuits and horizontal saccades with jerking head thrusting and nystagmus [7]. A related disorder has also been termed cerebello-oculorenal syndrome (CORS) $[4,8]$. Some cases of JBTS may be associated with nephronophthisis and retinal dystrophy or retinal coloboma [3]. To date, two underlying gene defects in patients with JBTS have been described. Homozygous NPHP1 deletions on chromosome 2q13 have been reported in two cases of JBTS associated with nephronophthisis [9]. However, it has been noted that NPHP1 deletions are not a common cause of JBTS [10]. More recently, mutations in AHII on chromosome 6q23.3 were described in patients with JBTS, but without any evidence of renal involvement $[11,12]$. In addition to these known gene defects, two loci have been mapped to $9 \mathrm{q} 34.3[13]$ and $11 \mathrm{p} 12-\mathrm{q} 13.3[14,15]$. Another putative locus was suggested by the detection of a chromosome $17 \mathrm{p} 11$ deletion in a patient with Smith-Magenis syndrome and a JBTS phenotype [16]. Here we report three novel mutations in AHII including, for the first time, the association of JBTS with NPHP. 
Fig. 1 Haplotyping and homozygosity mapping in F799. Paternal haplotypes are drawn to the left, and maternal haplotypes to the right. Markers on chromosome 6 ordered from top to bottom are centromeric to telomeric. Homozygous regions in each affected individual are boxed. The position of AHII is depicted by an arrow. Black symbols indicate individuals with affected status and white symbols indicate unaffected individuals; circles denote females and squares denote males

\section{F799}

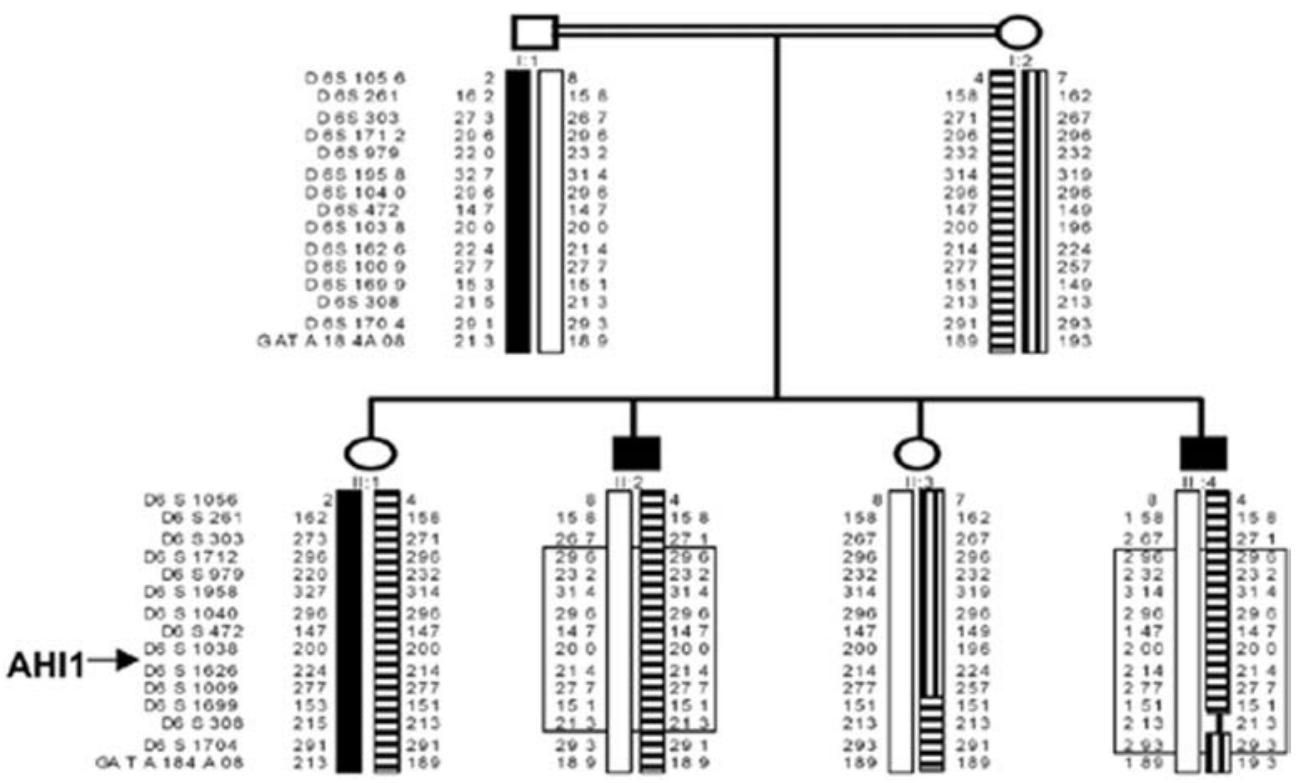

\section{Patients and methods}

Criteria for Joubert syndrome were based on the following clinical hallmarks: presence of cerebellar vermis aplasia, cerebellar ataxia, psychomotor developmental delay, nystagmus and oculomotor apraxia. The patients also had NPHP by clinical history and by renal biopsy in patient II-4 from family F799. Venous blood samples from family members were obtained after informed consent. Genomic DNA was prepared according to standard methods. NPHP1 deletions were excluded by Southern-blot analysis and haplotype analysis (data not shown). In four JBTS individuals from consanguineous families known to be homozygous for microsatellite markers across the AHII gene region [17], PCR of all coding sequences and the corresponding exon-intron boundaries of AHII where performed for mutational analysis. Primer sequences were determined using the UCSC sequence (http://genome.ucsc.edu/cgi-bin/hgGateway) and Primer3 software (http:// frodo.wi.mit.edu/cgi-bin/primer3/primer3_www.cgi). PCR products were purified (Marligen Biosciences) prior to direct sequencing (Genetic Analyzer 3700, Applied Biosystems). An additional 20 JBTS individuals from non-consanguineous families were also screened. Ninety-six healthy individuals were screened as negative controls for each novel AHII mutation.

\section{Results}

Homozygosity mapping in F799

Previous haplotyping data from a total genome search revealed four consanguineous families (F799, F700, F427 and F216) with JBTS that were homozygous at the AHII gene locus on chromosome 6q23.3 [17]. High-resolution haplotyping using 15 microsatellite markers was performed in these families. A region of homozygosity of more than $27 \mathrm{Mb}$ was confirmed in family F799, delimited by flanking markers D6S303 and D6S1704 (Fig. 1). However, regions of homozygosity were found to be interrupted by heterozygous markers in consanguineous families F700, F427 and F216, excluding them from this locus.

Mutational analysis of the AHII gene

Mutational analysis was performed by exon PCR covering all coding sequences and the corresponding exon-intron boundaries of the AHII gene in families F799, F700, F427 and F216. Exon PCR and direct sequencing in F799 revealed a homozygous single $\mathrm{T}$ nucleotide insertion (2369insT) within exon 16 in both affected siblings (II-2 and II-4) resulting in a frame shift and a subsequent stop codon at amino acid 791 (Fig. 2). Segregation of the mutation was demonstrated with the mother and unaffected siblings II-1 and II-3 showing a heterozygous T nucleotide insertion.

AHII mutations were not detected in families F700, F427 and F216. Direct sequencing confirmed a lack of homozygosity in these families, revealing heterozygous intronic single-nucleotide polymorphisms (SNPs) within sequencing PCR amplicons (data not shown). Additional screening for $A H I I$ mutations in 20 additional individuals with JBTS revealed two compound heterozygous mutations within exon 6 of AHII in family F400 (Fig. 2). The first mutation is a 324 heterozygous nucleotide deletion (365-688del) leading to an inframe deletion of 108 amino acids. The latter is a single A nucleotide insertion at position 444 leading to a frame shift and stop codon at amino acid 149. All mutations were absent from 96 healthy control individuals. 


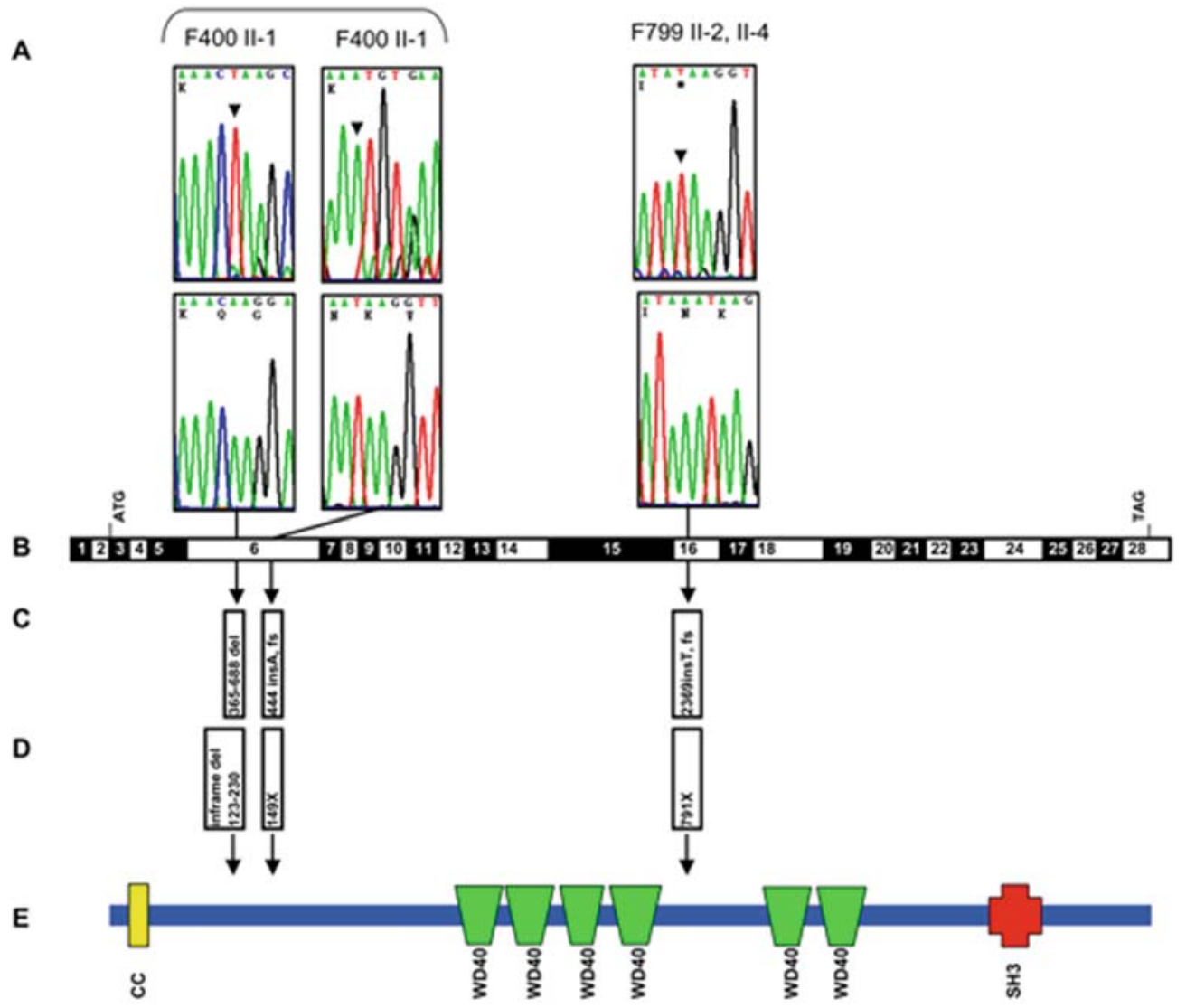

Fig. 2 Identification of three novel AHI1 mutations in patients with JBTS. A AHI1 mutations detected in F400 and F799 with JBTS. Sequence traces (nucleotides and respective codon) are shown for affected individuals (top) and healthy controls (below). Compound heterozygous mutations are marked with a bracket. *Denotes a premature stop codon. Note that double sequence in the mutated allele (444insA,fs) of F400 II-1 does not reflect the wild type allele since the other allele carries another mutation (365-688del) within

\section{Clinical features in JBTS patients with AHII mutations}

Family F799 originated from Pakistan. The affected individuals II-2 and II-4 were children of a consanguineous marriage whose parents were third degree cousins. The clinical features of both affected males in F799 included cerebellar ataxia, psychomotor developmental delay, nystagmus and oculomotor apraxia. The youngest sibling (II-4) had a salt and water-losing nephropathy leading to end-stage renal failure by the age of 16 years. Corticomedullary cysts were revealed on renal ultrasound, and a renal biopsy demonstrated changes consistent with nephronophthisis. The second affected sibling (II-2) had no renal symptoms, in particular no polyuria, and had not developed renal insufficiency by 20 years of age. However, this does not exclude a milder form of nephronophthisis in this patient. Siblings II-1 and II-3 remain healthy. Patient II-1 of family F400, of unknown ethnic origin, had JBTS associated with coloboma with no clinical evidence of nephronophthisis and no documented renal impairment. Additional clinical data, including renal ultrasound, were unavailable. the same exon. B Exon structure of human AHI1 cDNA. Positions of start codon $(A T G)$ in exon 3 and of stop codon $(T A G)$ in exon 28 are indicated. C Nucleotide changes and $(\mathbf{D})$ corresponding amino acid changes are shown in boxes. E Representations of AHI1 (Jouberin) protein motifs are drawn to scale in relation to exon structure. Positions of the mutations detected are indicated by $a r-$ rows. $C C$ coiled-coil domain; WD4O WD40 domain; SH3 src homology-3 domain

\section{Discussion}

We have detected, by direct mutational analysis in three patients, three novel mutations in AHII, including one patient with NPHP-associated JBTS, one patient with JBTS without renal involvement and one patient with JBTS associated with coloboma. AHII is highly expressed in human brain and kidney [12]. Within the brain, there is AHII mRNA expression in both the cerebellum and cerebral cortex [12] consistent with the marked cerebellar ataxia seen JBTS patients. The AHI1 protein, also known as Jouberin, possesses several distinct protein domains, which include one coiled-coil, six WD40 domains and one SH3 domain. Based on these domains, a role for Jouberin as an adapter protein for protein-protein interactions has been postulated [11].

Mutations in AHII have previously been described only in JBTS patients without a renal phenotype. Some of these patients had evidence of additional central nervous system abnormalities that included polymicrogyria [11]. These published mutations include two nonsense mutations, R351X and R435X, and a missense mutation 
V443D described by Ferland et al. [12]. The same missense mutation was also independently reported by Dixon-Salazar et al., in addition to two further frame shift mutations leading to truncations at codons 270 and 408 , respectively [11]. Thus, four truncating mutations were previously reported, leading to a loss of WD40 domains and SH3 domain regions at the C-terminus of the protein. All of these mutations were reported in JBTS patients without NPHP. Our report adds further genetic and clinical heterogeneity to patients with JBTS and AHII mutations, as the three novel mutations detected also lead to loss of both the SH3 domain and part or all of the WD40 domains in each case (Fig. 2B-E). It is of note that, like Jouberin, nephrocystin-1 has both an $\mathrm{SH} 3$ domain and a coiled-coil domain; therefore, it is likely that these proteins may interact within a functional complex both in the kidney and the brain. Homozygous mutations in NPHPI may sometimes lead to a JBTS phenotype, although this is rare. This implies that genotype and clinical phenotype correlations are difficult to make and that additional proteins within the nephrocystin-1/Jouberin complex may determine the phenotype via genetic modifiers. SH3 domains typically bind to PXXP amino acid motifs [18], and indeed the AHI1 protein contains four PXXP motifs. Therefore, since similar truncating mutations were found in JBTS patients with and without NPHP, the position of the truncation does not seem to explain the presence or absence of renal involvement. One potential explanation could be the action of additional "modifier" genes, analogous to the related disorder Bardet-Biedl syndrome, the clinical spectrum of which includes renal failure [19]. In Bardet-Biedl syndrome oligogenic mutations may potentiate a phenotype that is caused by two disease-causing recessive mutations in an independent gene [19].

In conclusion, AHII mutations are a cause of JBTS, and we demonstrate that novel truncating mutations are present in an affected individual with NPHP-associated JBTS. The role of AHI1 in renal cyst development and its interaction with other nephrocystins will require further investigation.

Acknowledgements The authors express their sincere appreciation to the patients and their families for their participation in this study. FH is the Frederick G.L. Huetwell Professor for the Cure and Prevention of Birth Defects and was supported by grants from the NIH, R01-DK068306-0 and R01-DK064614-02.

\section{References}

1. Joubert M, Eisenring JJ, Robb JP, Andermann F (1969) Familial agenesis of the cerebellar vermis. A syndrome of episodic hyperpnea, abnormal eye movements, ataxia, and retardation. Neurology 19:813-825

2. Boltshauser E (2002) Joubert syndrome: more than lower cerebellar vermis hypoplasia, less than a complex brain malformation. Am J Med Genet 109:332

3. Saraiva JM, Baraitser M (1992) Joubert syndrome: a review. Am J Med Genet 43:726-731

4. Gleeson JG, Keeler LC, Parisi MA, Marsh SE, Chance PF, Glass IA, Graham JM Jr, Maria BL, Barkovich AJ, Dobyns WB (2004) Molar tooth sign of the midbrain-hindbrain junction: occurrence in multiple distinct syndromes. Am J Med Genet 125A:125-134; discussion 117

5. Maria BL, Hoang KB, Tusa RJ, Mancuso AA, Hamed LM, Quisling RG, Hove MT, Fennell EB, Booth-Jones M, Ringdahl DM, Yachnis AT, Creel G, Frerking B (1997) "Joubert syndrome" revisited: key ocular motor signs with magnetic resonance imaging correlation. J Child Neurol 12:423-430

6. Maria BL, Quisling RG, Rosainz LC, Yachnis AT, Gitten J, Dede D, Fennell E (1999) Molar tooth sign in Joubert syndrome: clinical, radiological, and pathologic significance. J Child Neurol 14:368-376

7. Tusa RJ, Hove MT (1999) Ocular and oculomotor signs in Joubert syndrome. J Child Neurol 14:621-627

8. Valente EM, Marsh SE, Castori M, Dixon-Salazar T, Bertini E, Al-Gazali L, Messer J, Barbot Geoffrey Woods CG, Boltshauser E, Al-Tawari AA, Salpietro CD, Kayserili H, Sztriha L, Gribaa M, Koenig M, Dallapiccola B, Gleeson JG (2005) Distinguishing the four genetic causes of Joubert's syndromerelated disorders. Ann Neurol 57:513-519

9. Parisi MA, Bennett CL, Eckert ML, Dobyns WB, Gleeson JG, Shaw D, McDonald R, Eddy A, Chance PF, Glass IA (2004) The NPHP1 gene deletion associated with juvenile nephronophthisis is present in a subset of individuals with Joubert syndrome. Am J Hum Genet 75:82-91

10. Castori M, Valente EM, Donati MA, Salvi Fazzi E, Procopio E, Galluccio T, Emma F, Dallapiccola B, Bertini E, the Italian MTS Study Group (2005) NPHP1 gene deletion is a rare cause of Joubert syndrome related disorders. J Med Genet 42:e9

11. Dixon-Salazar T, Silhavy JL, Marsh SE, Louie CM, Scott LC, Gururaj A, Al-Gazali L, Al-Tawar AA, Kayserili H, Sztriha L, Gleeson JG (2004) Mutations in the AHII gene, encoding jouberin, cause Joubert syndrome with cortical polymicrogyria. Am J Hum Genet 75:979-987

12. Ferland RJ, Eyaid W, Collura RV, Tully LD, Hill RS, Al-Nouri D, Al Rumayyan A, Topcu M, Gascon G, Bodell A, Shugart YY, Ruvolo M, Walsh CA (2004) Abnormal cerebellar development and axonal decussation due to mutations in AHII in Joubert syndrome. Nat Genet 36:1008-1013

13. Saar K, Al-Gazali L, Sztriha L, Rueschendorf F, Nur-E-Kamal M, Reis A, Bayoumi R (1999) Homozygosity mapping in families with Joubert syndrome identifies a locus on chromosome $9 \mathrm{q} 34.3$ and evidence for genetic heterogeneity. Am J Hum Genet 65:1666-1671

14. Keeler LC, Marsh SE, Leeflang EP, Woods CG, Sztriha L, AlGazali L, Gururaj A, Gleeson JG (2003) Linkage analysis in families with Joubert syndrome plus oculo-renal involvement identifies the CORS2 locus on chromosome 11p12-q13.3. Am J Hum Genet 73:656-662

15. Valente EM, Salpietro DC, Brancati F, Bertini E, Galluccio T, Tortorella G, Briuglia S, Dallapiccola B (2003) Description, nomenclature, and mapping of a novel cerebello-renal syndrome with the molar tooth malformation. Am J Hum Genet 73:663-670

16. Natacci F, Corrado L, Pierri M, Rossetti M, Zuccarini C, Riva P, Miozzo M, Larizza L (2000) Patient with large 17p11.2 deletion presenting with Smith-Magenis syndrome and Joubert syndrome phenotype. Am J Med Genet 95:467-472

17. Utsch B, Attanasio M, Hennies HC, Pohl M, Omram H, Roos R, Kuehr J,Nauta J, Pereira R, Otto EA, and Hildebrandt F (2004) Confirmation and refinement of a gene locus (JBTS3) on chromosome 6q23.1-q23.3 for Joubert syndrome type 3 with renal involvement -exclusion of candidate genes. J Am Soc Nephrol 15:215A

18. Hildebrandt F, Otto E, Rensing C, Nothwang HG, Vollmer M Adolphus J, Hanusch H, Brandis M (1997) A novel gene encoding an $\mathrm{SH} 3$ domain protein is mutated in nephronophthisis type 1. Nat Genet 17:149-153

19. Badano JL, Kim JC, Hoskins BE, Lewis RA, Ansley SJ, Cutler DJ, Castellan C, Beales PL, Leroux MR, Katsanis N (2003) Heterozygous mutations in $B B S 1, B B S 2$ and $B B S 6$ have a potential epistatic effect on Bardet-Biedl patients with two mutations at a second BBS locus. Hum Mol Genet 12:1651-1659 